\author{
Nesrin Ozalp ${ }^{1}$ \\ Mechanical Engineering Department, \\ Sustainable Energy Research Laboratory, \\ Texas A\&M University at Qatar, \\ P.0. Box 23874 Doha, Qatar \\ e-mail: nesrin.ozalp@qatar.tamu.edu
}

\author{
Min-Hsiu Chien \\ e-mail: scottamm@neo.tamu.edu \\ Gerald Morrison \\ e-mail: gmorrison@tamu.edu \\ Mechanical Engineering Department,
Turbomachinery Laboratory,
Texas A\&M University,
College Station, TX 77840
}

\section{Computational Fluid Dynamics and Particle Image Velocimetry Characterization of a Solar Cyclone Reactor}

\begin{abstract}
Solar thermal cracking of methane produces two valuable products, hydrogen gas and solid carbon, both of which can be used as a fuel and as a commodity. During the course of this two-phase phenomenon, carbon particles tend to deposit on the solar reactor window, wall, and exit. When they accumulate at the reactor exit, the agglomeration of these particles completely blocks the exit. This problem has been the major issue preventing solar cracking reactors from running continuously. To address this problem, a cyclone solar reactor was designed to enhance the residence time and allow carbon particles to rotate in the reactor instead of moving towards the exit inlarge particle groups together. A prototype reactor was manufactured to test the concept, to better understand and explain the flow dynamics inside the solar cyclone reactor and to analyze the flow via particle image velocimetry (PIV). Advanced measurement and computational techniques were applied to build the prototype reactor. Computational fluid dynamics (CFD) analysis employing discrete phase model (DPM) was used to predict the particle transport phenomenadel (DPM), whereas $P I V$ was applied for the experimental part of the work. To understand the flow evolution along the vortex line, several images in the axial direction along the vortex line were captured. The results showed that when the main flow was increased by $25 \%$, the axial velocity components became larger. It was also observed that the vertical vortices along the vortex line showed stronger interaction with outward fluid in the core region. This implied that the horizontal twisting motion dominated the region due to the main flow, which could trap the particles in the reactor for a longer time. Furthermore, when the main flow was increased by $50 \%$, the flow displayed a cyclone-dominated structure. During the velocity evolution along the vortex line, more vortices emerged between the wall region and core region, implying that the energy was transferred from order to disorder. In summary, by appropriate selection of parameters, the concept of an aero-shielded solar cyclone reactor can be an attractive option to overcome the problem of carbon particle deposition at the reactor walls and exit. [DOI: 10.1115/1.4023183]
\end{abstract}

Keywords: solar reactor, carbon clogging, particle image velocimetry, CFD, flow dynamics

\section{Introduction}

Production of fuels and commodities without discharging hazardous wastes or emitting greenhouse or toxic gases is a pressing issue for many industries. Solar cracking of natural gas provides a very promising solution to petroleum, chemical and carbon industries by offering emission free production of hydrogen and carbon black, which are two essential products and feedstock in these industries depending on their need and the process $[1,2]$. For example, hydrocracking, hydrotreating, and hydroprocessing are essential processes in refining crude oil for the production of many products we use daily [3-5]. These processes consume large amounts of hydrogen making hydrogen one of the most important products in petroleum industry. In the same way, the chemical industry consumes hydrogen as a feedstock to make other commodities, such as ammonia. Finally, the carbon industry manufactures many types and grades of carbon for use in items such as car tires, batteries etc.

Current methods of hydrogen and carbon production practiced in industry emit large amounts of environmentally unfriendly

\footnotetext{
${ }^{1}$ Corresponding author.

Contributed by the Solar Energy Division of ASME for publication in the Journal of Solar ENERgy ENGINEERING. Manuscript received June 17, 2012; final manuscript received November 27, 2012; published online February 8, 2013. Assoc. Editor: Wojciech Lipinski.
}

gases into the atmosphere [2]. Therefore, solar cracking of natural gas can be considered as an alternative to the conventional way of hydrogen and carbon production to obtain these two valuable commodities in one process and without emitting any hazardous gases via the following reaction.

$$
\mathrm{CH}_{4} \rightarrow 2 \mathrm{H}_{2}+\mathrm{C}(\text { solid }) \quad \Delta \mathrm{H}_{298}=75.6 \mathrm{~kJ} / \mathrm{mol}
$$

This endothermic reaction receives the necessary high temperature process heat from concentrated solar energy in a solar reactor, where the reaction takes place.

Solar cracking is an emission free process, in contrast to traditional steam reforming of natural gas where chemical bonds between hydrogen and carbon are broken via steam. In spite of it is environmentally attractiveness, this process faces challenges like protecting the window from carbon particles, reactor clogging due to carbon particle deposition, protection of the reactor against thermal shocks and loss of solar power from the window due to multiple reflections. There have been many innovative solar cracking reactor designs aimed at achieving increased conversion efficiencies through novel flows from vortex-flow to tornado, and from fluidized bed to rotating cavity [6-12]. The designs of these solar reactors seek enhanced flow conditions that result in improved overall efficiencies and some reduction in carbon deposition, but the carbon deposition at the reactor exit remains. This 
paper summarizes the design process of the solar cyclone reactor concept as a solution to this problem, and provides the results of a PIV analysis on the flow dynamics of this reactor concept.

\section{Design of the Solar Cyclone Reactor}

It is important to understand how the carbon particles move inside the solar reactor so that a solution to prevent them from clogging the reactor exit can be found. Figure 1 illustrates this process, where natural gas enters into a solar reactor and the decomposition products, hydrogen gas and solid carbon particles move toward the exit. On the way toward the exit, some of the carbon particles deposit on the reactor window and walls, while the remaining carbon particles collectively migrate towards the exit. Because of this collective particle motion, the volume of carbon particles exiting the reactor causes clogging. This also blocks the passage for hydrogen and undecomposed natural gas.

Exit blockage increases the reactor pressure which eventually breaks the quartz window. Therefore, preventing the collective carbon particle movement toward the reactor exit is essential. This can be achieved by increasing the residence time and by making the carbon particles rotate inside the reactor before moving toward the exit, via use of a catalyst or by adjusting the flow dynamics to serve the purpose. It should be noted that the goal is to produce as much hydrogen and carbon as possible by collecting them after the exit. However, if the carbon particles deposit inside the reactor or if they block the exit, then the production of these valuable commodities is terminated.

The carbon deposition on the window has been tackled by injecting inert gas flow to sweep the window [13,14], however carbon deposition on the reactor walls still remains unsolved. Therefore, a new solar reactor concept was designed at Texas A\&M University at Qatar to address these two remaining problems by reducing carbon deposition on the walls and at the exit.

During the design process of our solar cyclone reactor, these two problems were addressed by studying each of them at a time starting from the carbon deposition at the exit. To increase the residence time and to make carbon particles complete several turns inside the reactor before they exit; a cyclone flow in the center was created via iterative design of the inlet ports, e.g., identifying the optimum location, injection angle, velocity etc. Iterations were made based on CFD simulation results. The first design achieved a cyclone of required characteristics at the required swirling effect, included 6 inlet jets for the main flow [15]. Simulations to identify the best location and the position of these jet ports and natural gas velocity resulted with a gas velocity of $45 \mathrm{deg}$ angle and $6 \mathrm{~m} / \mathrm{s}$. Figure 2 illustrates one of the initial successful designs.

The first step of the reactor design process was to validate the CFD results by running the code to simulate the reactor designs of other research groups and to compare the simulation results with the published experimental results of those research groups. For instance, flow behavior and thermal interaction of gas-particle flow for a directly irradiated vortex flow solar reactor of Ref. [14] presents a good example to study solar cracking process computationally. To do this, the thermal hydraulics between gas flow and particle were studied by a two way coupled Euler-Lagrange approach. A two band discrete ordinate model was considered to solve radiative transport between walls and entrained particles. The effect of main flow, secondary flow, particle loading, particle diameter, and residence time were studied to analyze flow physics and heat transfer. The static temperature contours, temperature distribution along the center line of the cavity, path lines and particle temperatures were obtained. The numerical simulations predicted the mixture (gas-particle) flow outlet and cavity wall temperatures of the experimentally measured values of Ref. [14] by $3.59 \%$ and $9.38 \%$ different experimentally measured values [16]. We also checked the validation of the CFD simulations against the experimental observations of Ref. [17], where carbon deposition was experimentally investigated in detail. The re-normalization group model (RNG) $k-\varepsilon$ model was used to evaluate turbulent quantities. Trajectories of the carbon particles and tracking them through the continuous phase of gas were done by employing a discrete phase model (DPM). The dispersion of particles due to turbulence in the fluid phase was predicted using a stochastic tracking model. In the discrete random walk (DRW) model, the fluctuating velocity components were sampled assuming a Gaussian probability distribution. The DRW results showed the effect of instantaneous velocities of gas flow field while the turbulent effects were considered. A DPM was used to evaluate the trajectories of the carbon particles and to track them through the continuous gas phase. As seen in Ref. [18], the CFD simulations successfully predicted the experimental observations of Ref. [17]. With confidence in that CFD code, it was applied to the reactor concept developed at Texas A\&M University at Qatar and used during the design iteration to investigate the impact of each change on the temperature distribution, flow dynamics, and carbon deposition. Figures 3-5 show the CFD simulation results of the solar reactor design given in Fig. 3.

In this reactor concept, carbon particles follow the cyclone path in the center however, some of them escape from the cyclone and deposit on the walls. To prevent this, a "wall screening" covering the walls like a cage was added, sweeping the escaped carbon particles off. Figure 4 shows this laminar flow which acts as an aeroshield to protect the wall from carbon deposition. A CFD simulation was performed in order to obtain a configuration where the laminar wall screening was not disturbed by the cyclone flow in the center.

Figure 5 shows the isometric view of the laminar flow and the cyclone flow co-existing without disturbing the laminar wall screening. However, in order to keep the laminar character of the wall screening flow, the Reynolds number was decreased which was a compromise from the turbulence. This initiated the

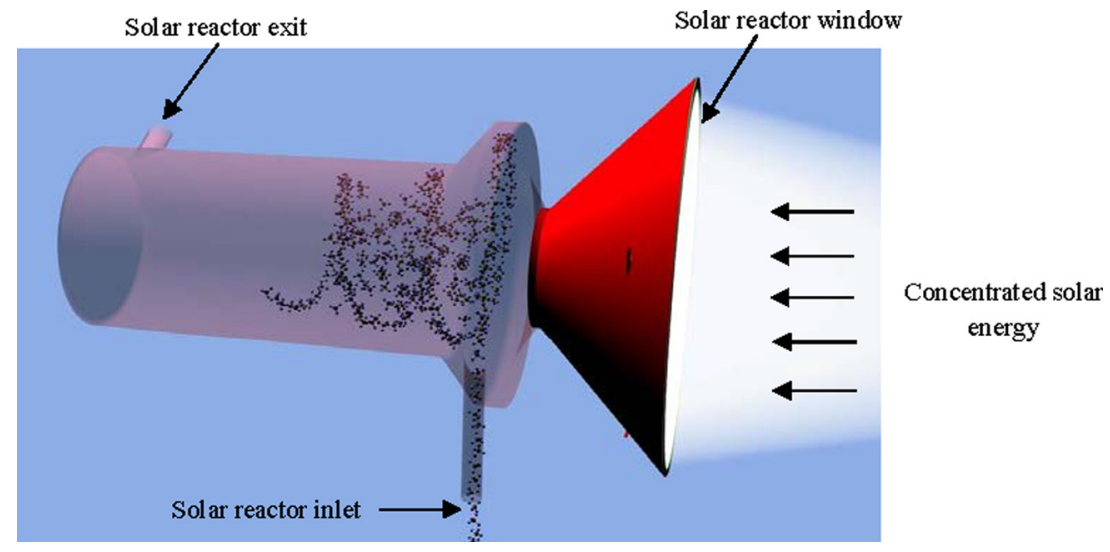

Fig. 1 Natural gas entry and product movement inside a solar reactor 


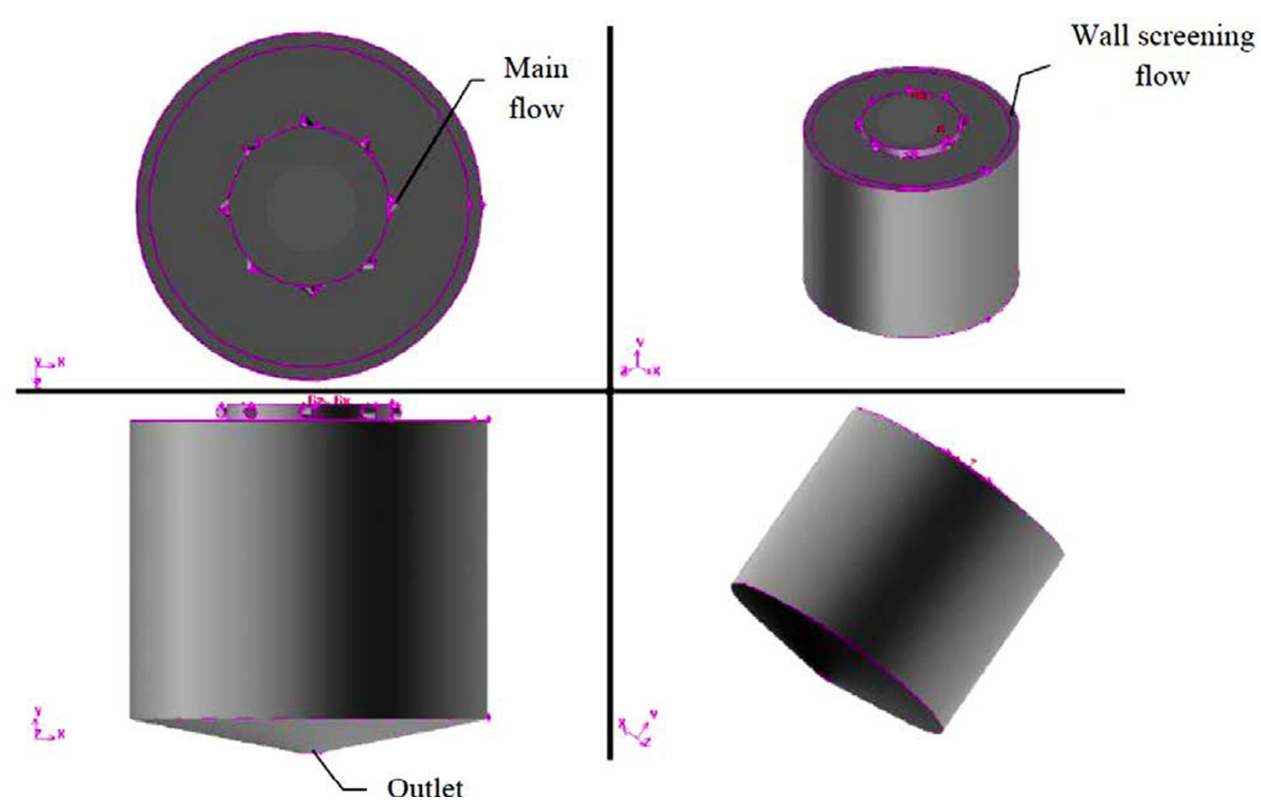

Fig. 2 One of the first solar cyclone reactor design concepts housing a successful cyclone in the center [15]

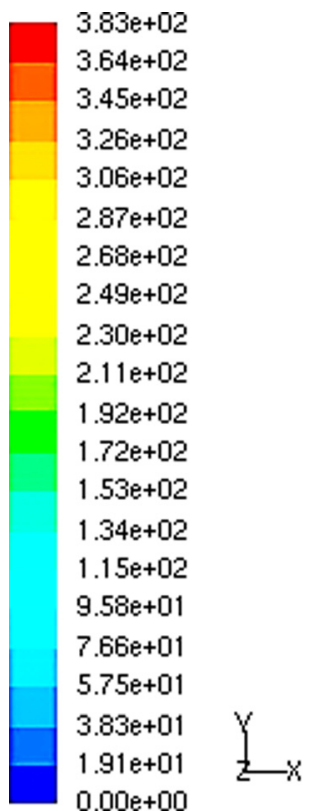

Fig. 3 Vortex flow inside the solar reactor [19]

development of the design shown in Fig. 2 to increase the turbulence effect but to maintain the laminar wall screening. The final result of that design development effort is illustrated in Fig. 6.

In this new design concept, "guide vanes" were added on the walls to allow wall screening flow through these channels. This geometry enhanced of the cyclone in the center and kept the laminar flow undisturbed.

The final solar reactor design, which is shown in Fig. 7, achieved a much stronger cyclone and the simulations on the carbon deposition showed that the wall screening did not allow carbon deposition on the walls and deposition at the exit was significantly reduced [20].

It can be seen from Figs. 2, 6, and 7 that the reactor design has significant impact on flow dynamics. The flow dynamics of the cyclone flow and the wall screening of this new reactor concept are illustrated in Fig. 8.
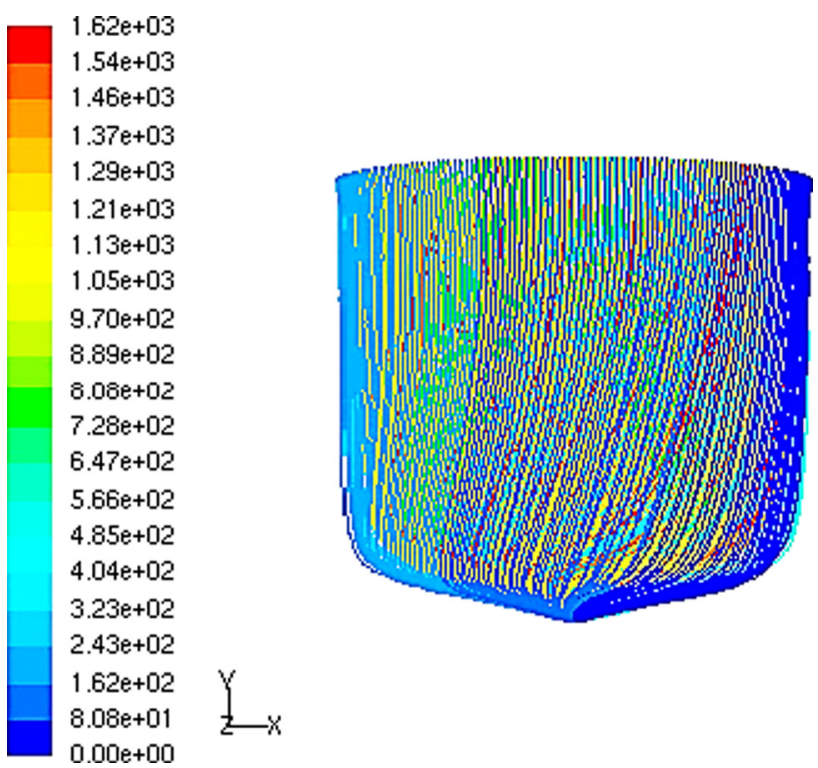

Fig. 4 Aero-shield on the solar reactor walls [19]

Figure 8 shows that the type of wall screening gas makes a difference in flow pattern. For example, in $(a)$, hydrogen is used as the wall screening gas which is completely disturbed by the cyclone flow. On the other hand, argon wall screening is not disturbed by the cyclone. In a similar way, Fig. 9 shows that argon wall screening gives more uniformed temperature distribution. Argon's ability to maintain a stable screening flow field compared to hydrogen might be explained due to its being heavier than hydrogen. Referring to the flow pattern inside the reactor for these two cases, it is observed that hydrogen is drawn into the cyclone leaving the walls unprotected while argon stands with no disruption. This impact is reflected on the temperature distribution with inhomogeneity showing that follow dynamics is indeed linked to heat transfer and eventual temperature distribution inside the reactor.

As for the carbon deposition, referring to Fig. 10, it is seen that carbon deposition on the walls was eliminated when argon was used as the wall screening gas. Figure 10 also shows that carbon 


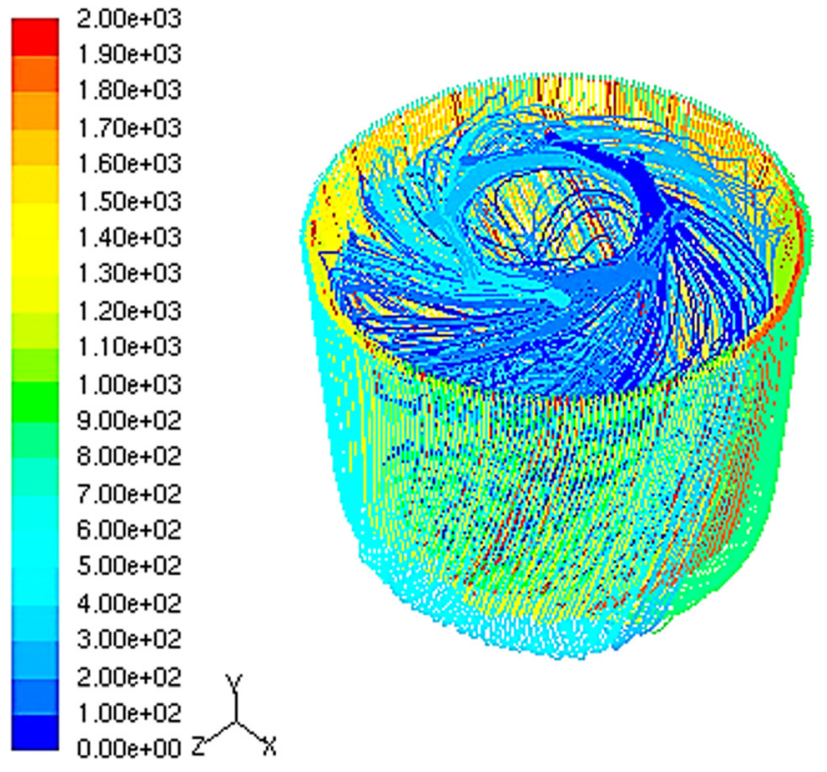

Fig. 5 Isometric view of the aero-shielded solar cyclone reactor [19]

deposition at the exit was significantly reduced with this reactor design.

In order to experimentally test this reactor concept and to further improve carbon particle tracking, a particle image velocimetry analysis was conducted at the Turbomachinery Laboratory of the Texas A\&M University in College Station, which is described in Particle Image Velocimetry Analysis of Solar Cyclone Reactor section.

\section{Particle Image Velocimetry Analysis of Solar Cyclone Reactor}

Based on the previous CFD results, an experiment was designed to validate the performance of the solar cyclone reactor designed by CFD. The main goal was to duplicate the CFD results experimentally by duplicating a flow field where the fluid dynamics is same as that in the solar cyclone reactor design. Once this was achieved, the next goal was to determine the optimal operating configuration by manipulating the inlet conditions to reduce the particle deposition. The flow control system and the experimental setup are given in Figs. 11 and 12, respectively. Air was used as the single source of three inlet gases for this study to reduce the complexity of the flow field setup. The source air was branched into three streams used to supply the main flow, the window screen flow, and the wall screen flow. The volumetric flow rate, pressure and temperature of the three flows were controlled, respectively, before entering the reactor. Downstream of the reactor, the pressure was regulated before discharging to the atmosphere. The seeding particles for the PIV measurement were injected into the source air flow before branching into the three streams.

To duplicate the CFD simulated flow field, the geometry of the solar cyclone reactor containing three flow inlets and a single outlet was manufactured. The reactor was constructed of polycarbonate which allowed optical access. Three beam blocks shown in Fig. 12 were placed on the path of the reflecting light to prevent reflecting light from entering into the optical cavity and to provide additional indication of light sheet alignment. The alignment between light sheet and the reactor was adjusted by turning bolts at the four screw legs of the base plate of the reactor. Figure 13 shows the geometry and the layout of the internal channels of the reactor.

The three gas streams enter the reactor via three inlets at the top of the reactor and discharge through the converging exit at the bottom. The main flow enters the reactor at a speed of $2.95 \mathrm{~m} / \mathrm{s}$ via 18 radial channels with a $45 \mathrm{deg}$ angle to the inner wall, forming a cyclone structure around the axis of the reactor. The two secondary flows act as screening and buffering fluids to protect the window and the inner reactor wall from particle deposition. The window screening flow enters the reactor at a speed of $0.33 \mathrm{~m} / \mathrm{s}$ radially via the annular clearance. The wall screening flow enters the reactor at a speed of $2.13 \mathrm{~m} / \mathrm{s}$ vertically via the ring-shaped clearance attached to the inner wall. Both clearances are $0.2 \mathrm{~mm}$ in width. Each clearance is connected to a buffering space which is $10 \mathrm{~mm}$ in width and $2 \mathrm{~mm}$ in height, located in the reactor which allows the flows to be fully mixed with seeding particles. Another buffer space is also used for the main flow before branching into the 18 jets. The total volumetric flow rate is $21 \mathrm{LPM}$ with 10 LPM for the main flow, 10 LPM for the wall screening flow and $1 \mathrm{LPM}$ for the window screening flow.

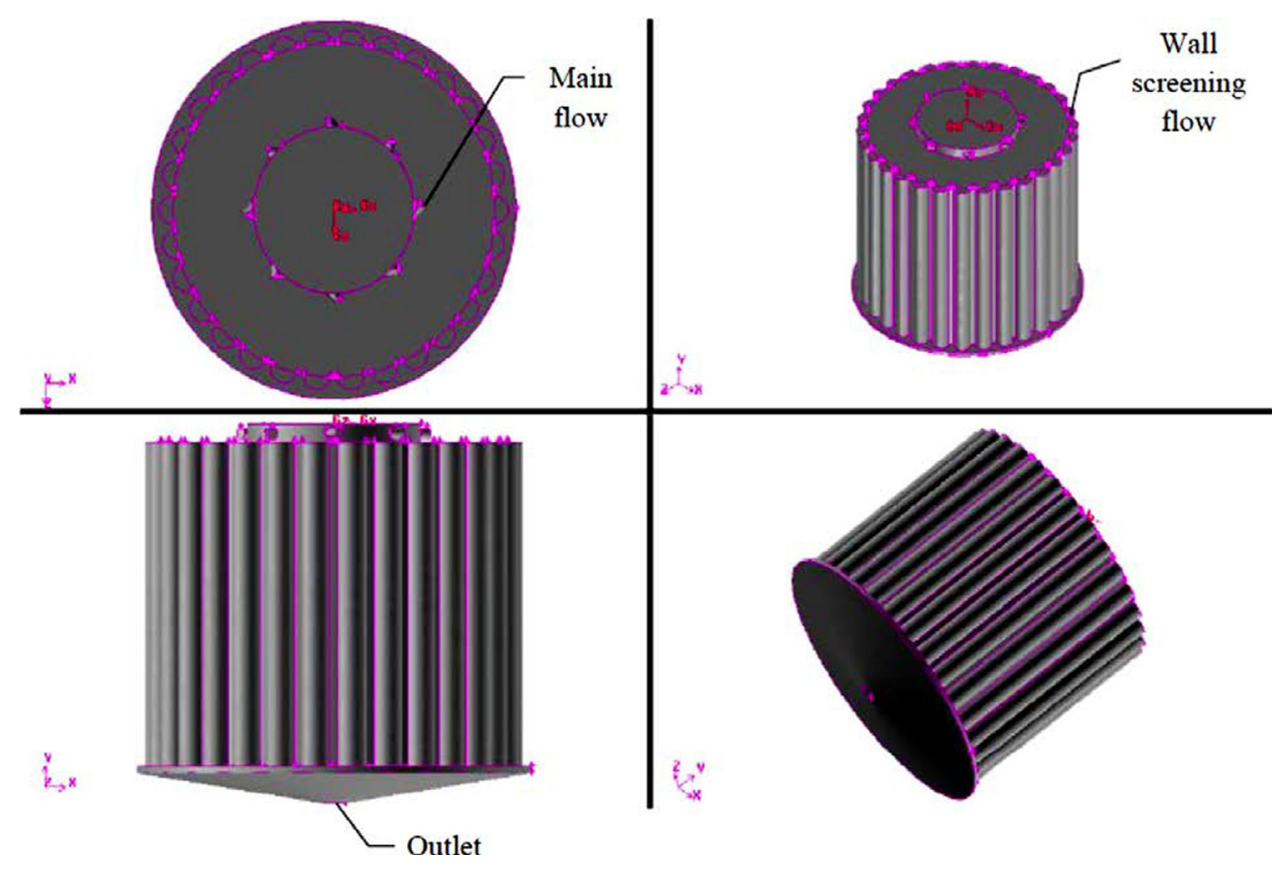

Fig. 6 Solar cyclone reactor with guided vanes 


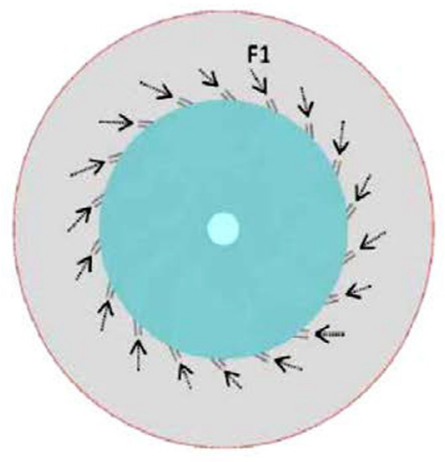

(a)

F1 Multiple flow inlets for methane

F2 Window screening $\left(\mathrm{H}_{2}\right)$

F3 Wall screening $\left(\mathrm{Ar} / \mathrm{H}_{2}\right)$

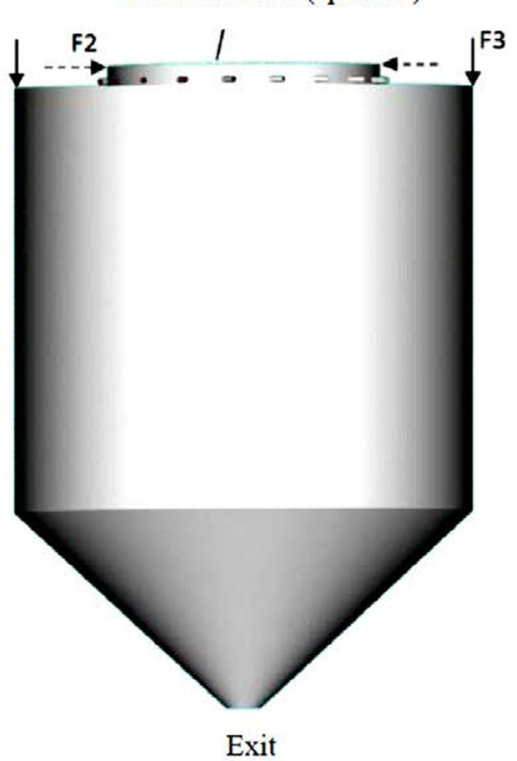

(b)

Fig. 7 Solar cyclone reactor with aero-shielded wall screening flow (a) top view and (b) front view [20]

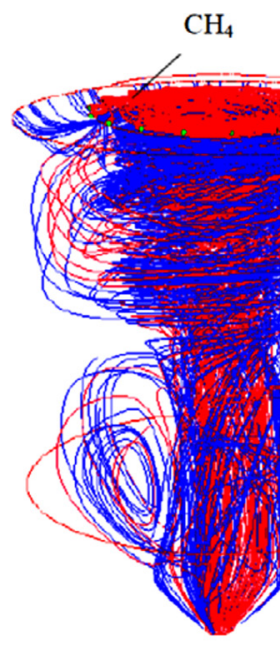

(a)
$\mathrm{H}_{2}$ (blue)

F2 Window screening (H2) (1 1/min)

F3 Wall screening $(\mathrm{Ar} / \mathrm{H} 2)(10 \mathrm{l} / \mathrm{min})$

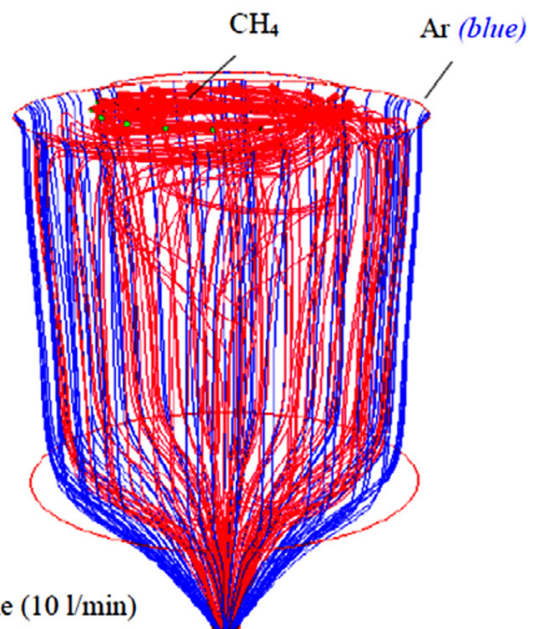

(b)

Fig. 8 Pathlines of the solar cyclone reactor with different wall screening flows (a) hydrogen and $(b)$ argon

Considering the velocity range and accessibility of measuring a turbulent flow field, the PIV technique is preferred because it provides nonintrusive measuring advantages. The advantages of nonintrusive and whole field measurement also makes PIV suitable for performing experiments in inaccessible or enclosed environments such as high temperature or active chemical reactions. In the development of the PIV technique in the past twenty years, Keane and Adrian [21,22] presented principles to follow in order to obtain images with unbiased information. The maximum particle displacement of in-plane and out-of-plane motions should not exceed one-quarter of the interrogation region size and the thickness of the light sheet, respectively, to avoid loss of particle image pairs which result in a rise of noise in image correlation.
In order to perform a PIV measurement, the entire reactor was built of transparent polycarbonate disk parts. The experiments were performed at room temperature. The volumetric flow rates of the three flows used in the CFD simulations were maintained in the experiment by adjusting the inlet conditions.

\section{Experimental Methodology and the Results}

In the experiment setup shown in Fig. 12, the measuring volume was illuminated by $532 \mathrm{~nm}$ Nd-YAG double-pulsed laser light sheet with $120 \mathrm{~mJ}$ at maximum in each pulse. The thickness of the light sheet was $1 \mathrm{~mm}$. The images of the flow field were captured by a 12 bit $4 \mathrm{M}$ pixel camera, synchronized with the laser 


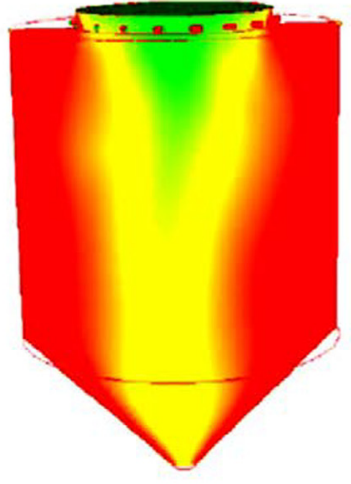

(a)

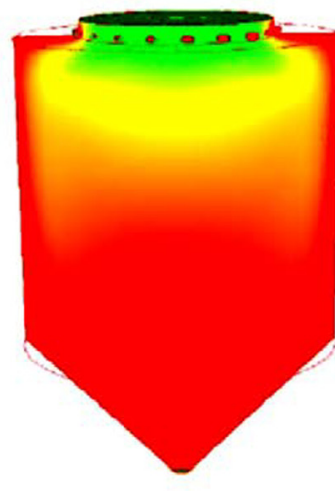

(b)
Temperature (K)

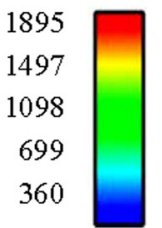

Fig. 9 Temperature contours of the solar cyclone reactor with different wall screening gas (a) hydrogen and $(b)$ argon

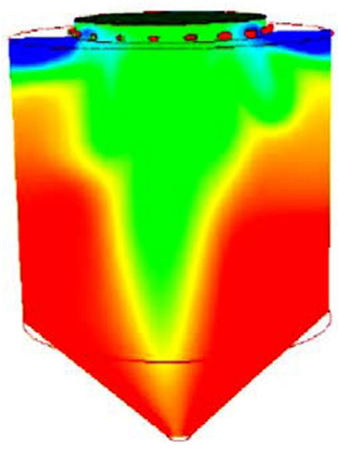

(a)

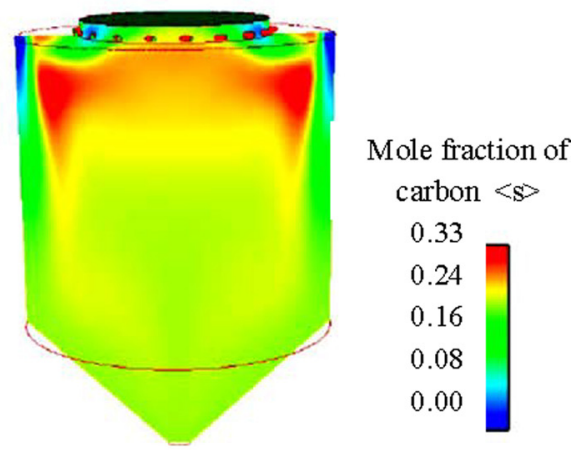

(b)

Fig. 10 Contours of carbon mole fraction with different wall screening gas (a) hydrogen and (b) argon

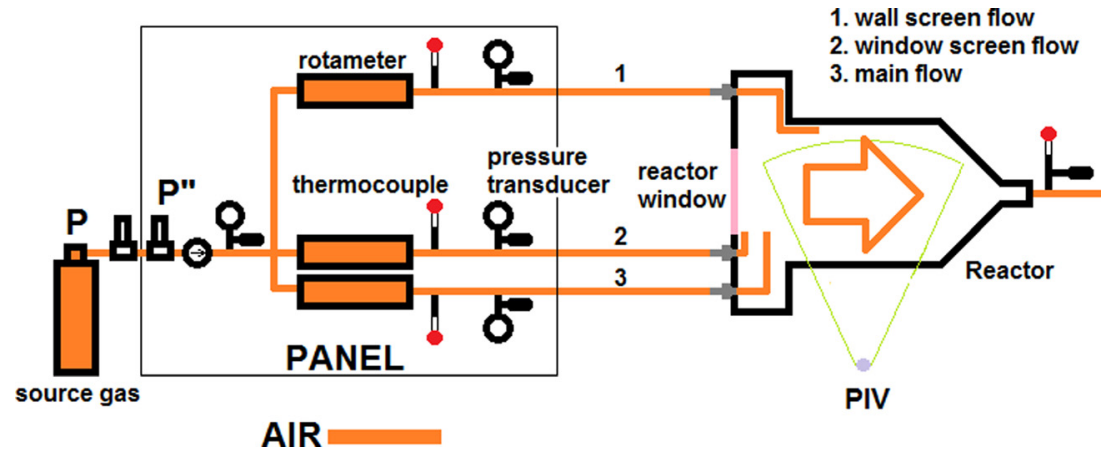

Fig. 11 Schematic of flow control system

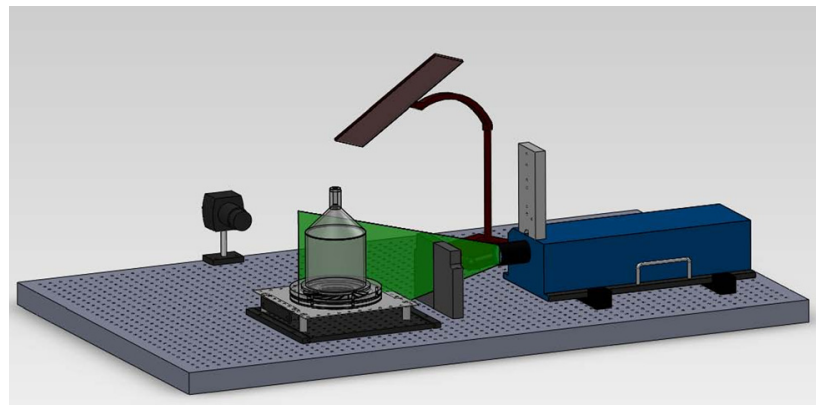

Fig. 12 Experimental setup of PIV by an external synchronizer. The seeding particles were supplied by an atomizer generating tri-ethylene glycol (TEG) particles $1 \mu \mathrm{m}$ in size. The PIV particle images were processed by using cross-correlation. Both multipassing and Rohaly-Hart correlations [23] were utilized in the image processing. The time interval in each pair of frames was estimated to be between 63 and $146 \mu \mathrm{s}$, according to the interrogation spot size and fluid flow conditions obtained from the CFD prediction. A mean test was performed in post processing under the spot size setting. The images were recorded at steady state on the basis of formation of a clear vortex line after a period of operating time under fixed input parameters. No vector filling or conditioning was added during the image processing. 


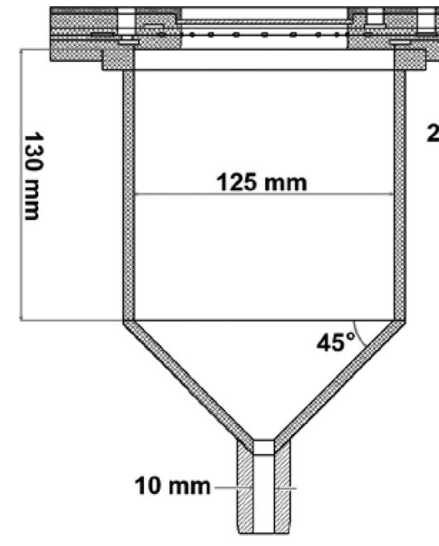

(a)

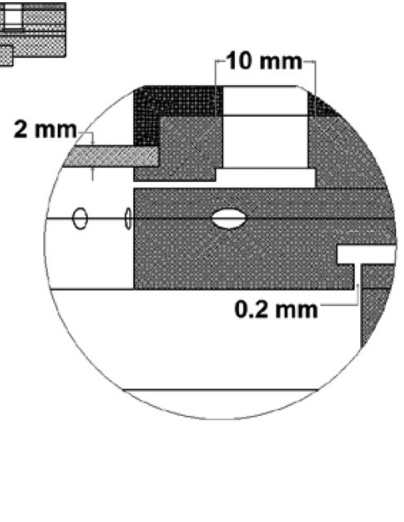

(b)

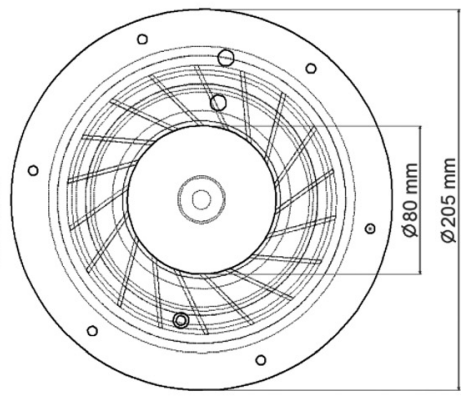

(c)

Fig. 13 Geometry of solar reactor (a) cross-sectional view, (b) internal channels of three inlet flows, and $(c)$ top view of reactor

The first step of the experiment was to choose a suitable seeding condition. The seeding concentration is dependent upon the supply pressure and the number of atomizer jets used in the atomizer. The concentration of the seed particles increases as the jet number and the supply pressure increases. It is also one of the most critical parameters that affect the overall quality of the image captured. In the experiments, two jets on a supply pressure of 25 psig were used to create an acceptable seeding concentration distributed across entire light sheet plane. An evaluation of the optical system as outlined in Ref. [22], the diameter of the selected seeding particles provided a magnification factor which was an order of magnitude smaller than the diffraction limit of the system lens, implying the seeding particles was small enough so as to not affecting the optical resolution. Although being suitable for PIV measurement, the seeding particles made of TEG are different from the carbon particles in the dissociation reaction. Fortunately, it was noted that the size of the carbon particle in the CFD prediction was in nanoscale. The Stoke's number of the carbon particles was found to be much smaller than that of seeding particles. Since the Stoke's number of a seeding particle was about 0.005 , this implies that the liquid particle's trajectory was the same as that of a carbon particle. Therefore, when the fluid dynamics was determined, the carbon particle paths were estimated.

The second step of the experiment was to determine the time interval in each image pair. The range of time intervals tested was $500 \mu \mathrm{s}$ and below. The processed images showed a tendency for the vector field to merge from the wall region toward the axis of the reactor as time interval decreased. When the time interval was equal to $500 \mu \mathrm{s}$, the velocities vectors near the wall were captured. However, the regions around the axis of the reactor were blank with no vectors covered due to active out-of-plane flow motions near the vortex line. As the time interval decreased, the velocity vectors around the vortex line began to be resolved. When the time interval was equal to $150 \mu \mathrm{s}$, the vector distributions on both sides of vortex line resolved. Below $150 \mu \mathrm{s}$, the vector distribution became independent of time interval.

Light distortion and reflection are critical issues when performing the optical measurements inside the transparent reactor due to reactor wall's thickness and curvature. The light distortion came from the difference of refractive indices between gas and solid interface and thickness of geometry. The quantification of light distortion through the cylindrical wall is presented in Fig. 14.

In Fig. 14, the light sheet paths were calculated at six incident positions in the $\mathrm{z}$ direction, which is the direction of traverse. The incident positions were separated by 0.5 in. The black solid contour represented the outer surface of the cylindrical wall; the dashed contour represented the inner surface of the cylindrical wall. The lines above the curve are incident light sheets and the lines below

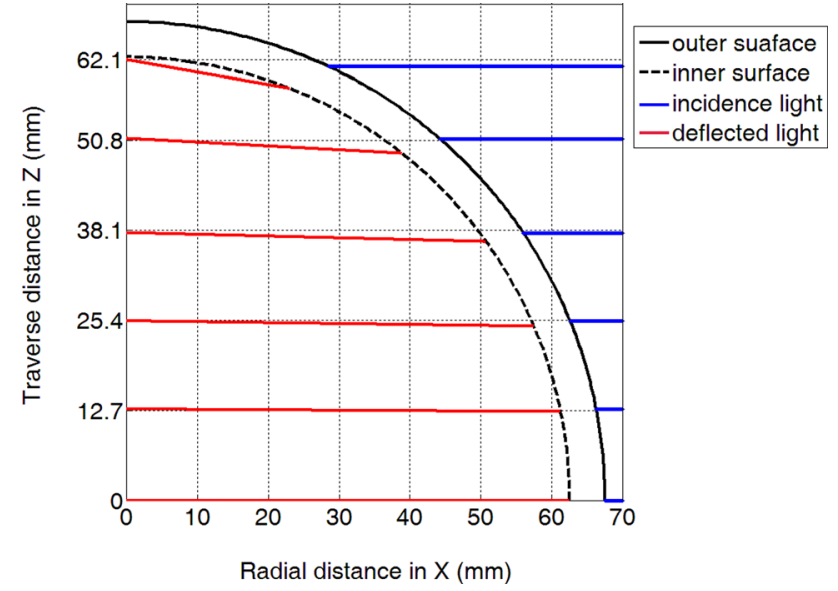

Fig. 14 Light distortion across cylinder wall

the dotted curve show the light paths corresponding to the incident positions. It was found that the distances of separation between different incident positions were maintained after refractions. According to Fig. 14, light deflection becomes significant in the fourth light path counted from the bottom. When the incident light sheet is traversed to and beyond $62.1 \mathrm{~mm}$ in $\mathrm{z}$ direction, corresponding to $68 \mathrm{deg}$ with respect to the origin, the total reflection occurs.

According to the light deflection analysis, the level of distortion became significant when the light sheet deviated from the axis of the reactor by more than 1 in. Thus, the images were captured at three locations which were $0,12.7$, and $25.4 \mathrm{~mm}$ deviated from the axis, respectively, under the distortion limit while considering the depth of field of the camera. The light reflected from a solid surface generates eight to nine pairs of bright vertical stripes on the wall where particle information is lost due to strong light intensity of the spectral reflections covering the area. One way to minimize this affect is to paint a laser absorbing dye on the inner surface where reflection occurred. The paint used absorbs the $532 \mathrm{~nm}$ laser light and re-emitted $650 \mathrm{~nm}$ light that can be filtered out by mounting a band pass filter on the camera lens.

Figure 15 shows contours of the velocity magnitude and vorticity in the measurement planes at the three traverse positions, 0 , 12.7 , and $25.4 \mathrm{~mm}$ from the axis of reactor. When the vortex line of the flow field is not on the light sheet plane, the tangential components of velocity are captured. Those tangential components form patterns of changing vortex directions shown in Fig. 15. The appearance of the vortex patterns indicates misalignment between the light sheet and the vortex line. During image capture of the 

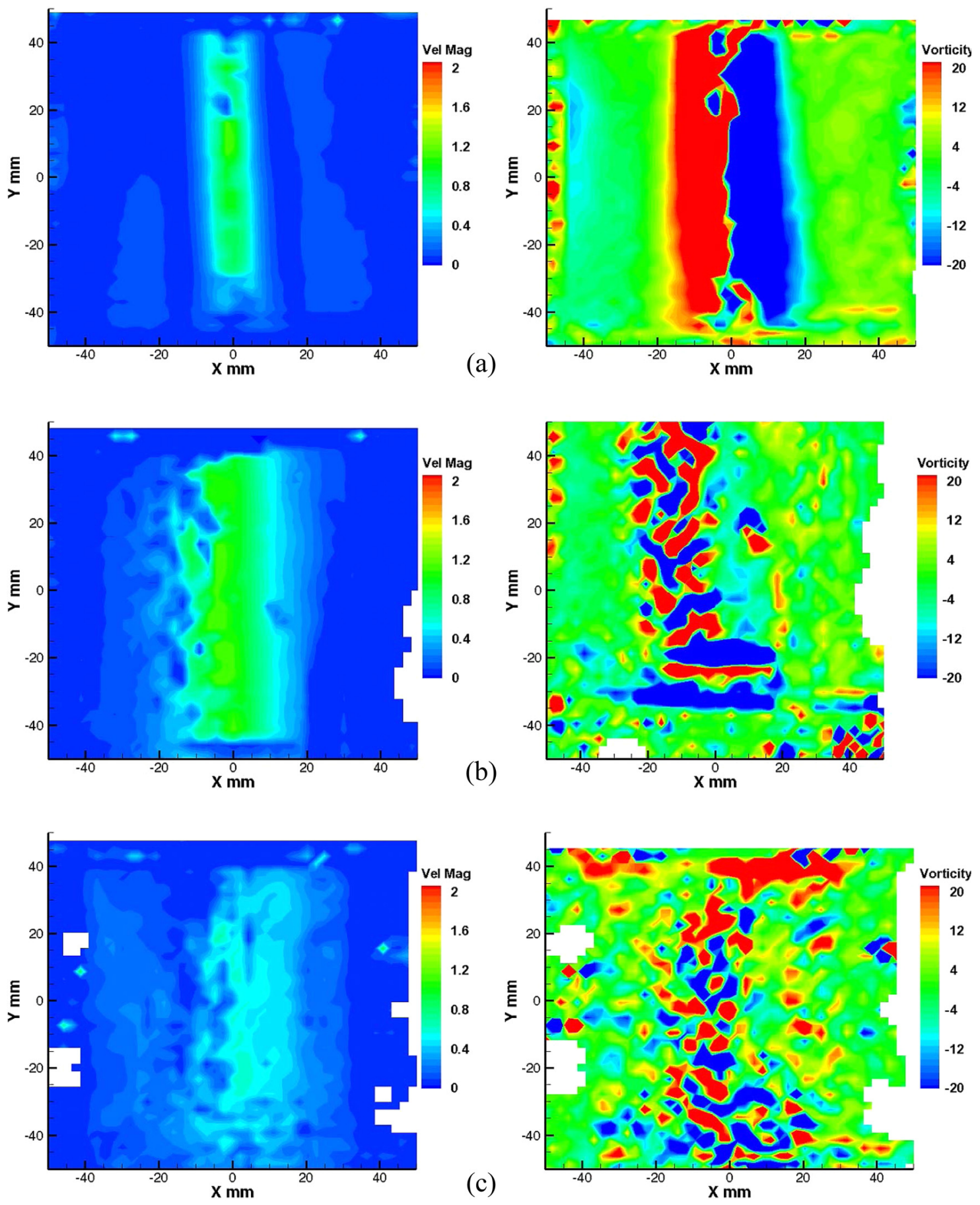

Fig. 15 Averaged magnitude contours of axial velocity and corresponding vortices on light sheet planes deviated from the aligned plane by: (a) $0 \mathrm{~mm}$ (aligned), (b) $12.7 \mathrm{~mm}$, and (c) $25.4 \mathrm{~mm}$

flow field, the vortex line precesses around the axis of the reactor. Thus the results should be obtained from the ensemble averaged field. When the light sheet was aligned with the vortex line statistically, the averaged result showed that the horizontal vortex strips vanished. Only a few residual of vortices which are at least one order smaller and more close to the bottom of the reactor remained. The averaged velocity vector field image showed that the axis-symmetric upward-moving flow along the vortex line in the core region dominated the vertical flow motion while those everywhere else were negligible in the light sheet plane. Due to the lack of tangential components and velocity averaging, the result showed blank area near the wall. Velocity vectors at bottom of the reactor were difficult to obtain since the region was dominated by flow motion perpendicular to the light sheet plane.
During the image capture, a drifting vortex line was observed. Along the vortex line a series of local scale vortices indicated an upward-moving flow with the highest velocity in the core region decaying toward the wall. It was also observed that the flow at the bottom of the reactor is dominated by the main flow which does not allow the particle displacements to be captured via PIV. Only a limited amount of seeding particles moving upward in the light sheet plane were captured, representing the minor component of in-plane motion compared to dominant component of twisting motions perpendicular to the light sheet plane. There was no significant global mean flow direction between the core and wall regions on the light sheet plane. Also, within this region on the light sheet plane, local motions are identified by only small particle displacements, implying a primary out-of-plane motion, which 
are referred as the particle motions perpendicular to the light sheet.

Contours of the vorticity along the axis of the reactor illustrate the flow field, which shows stronger vortices around the vortex line in the core region with minor vortices near the wall region. The vortices in the core region were induced by the interaction of fluid between the vortex core and the region around it. This suggests that the flow between vortex core and wall region are moving relatively slowly or even in the opposite direction to the fluid in the vortex core. The vortices near the wall region were the result of the wall
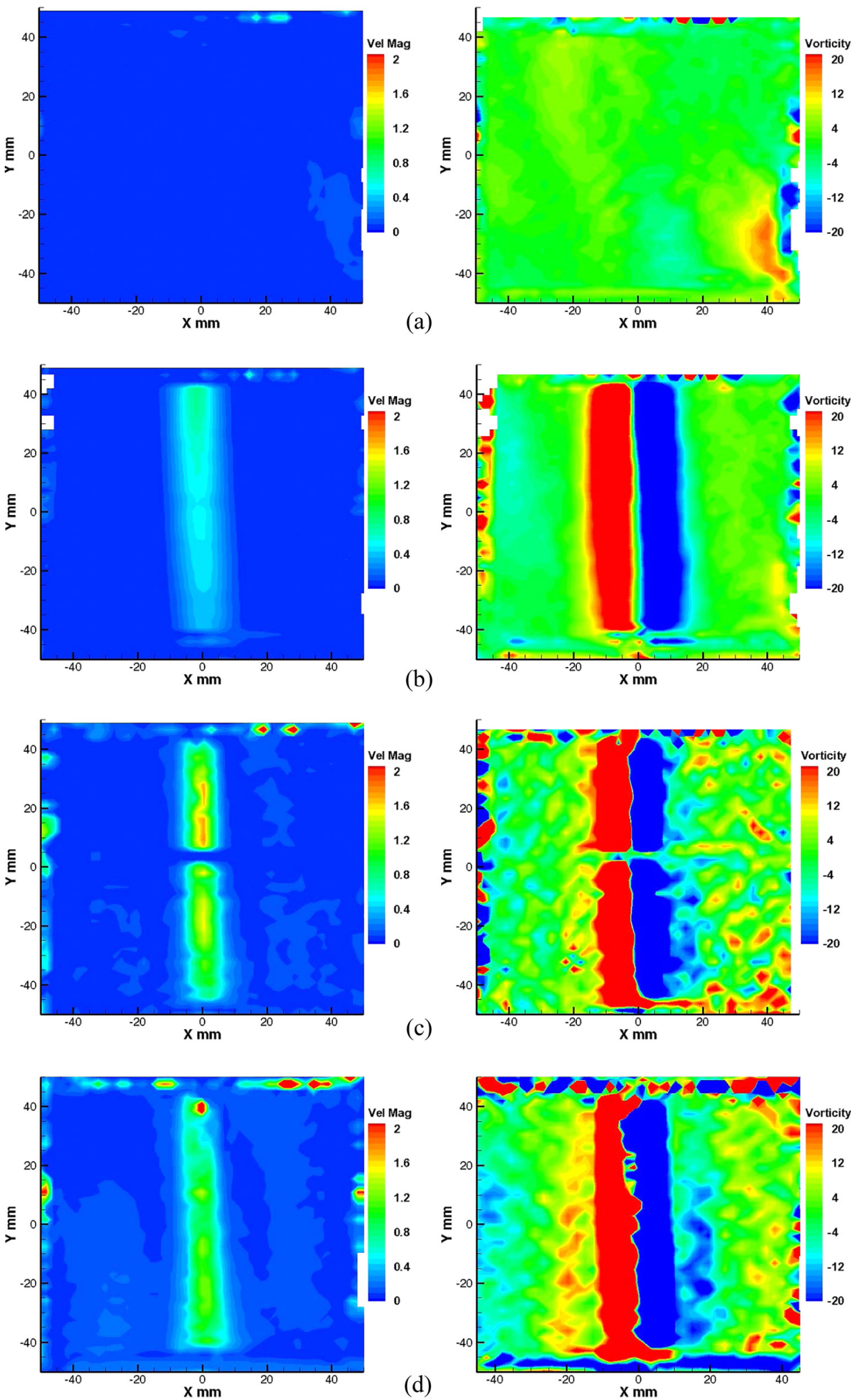

Fig. 16 Averaged magnitude contours of axial velocity $(\mathrm{m} / \mathrm{s})$ and corresponding vortices, according to different main flow rate with respect to CFD settings: (a) $50 \%$, (b) $75 \%,(c) 125 \%$, and (d) $150 \%$ 
screen flow and secondary flow, which can be identified by the vortices direction. It was also found that in the averaged field, the vortices between the core region and wall region vanished after vector field averaging, implying a local random flow nature.
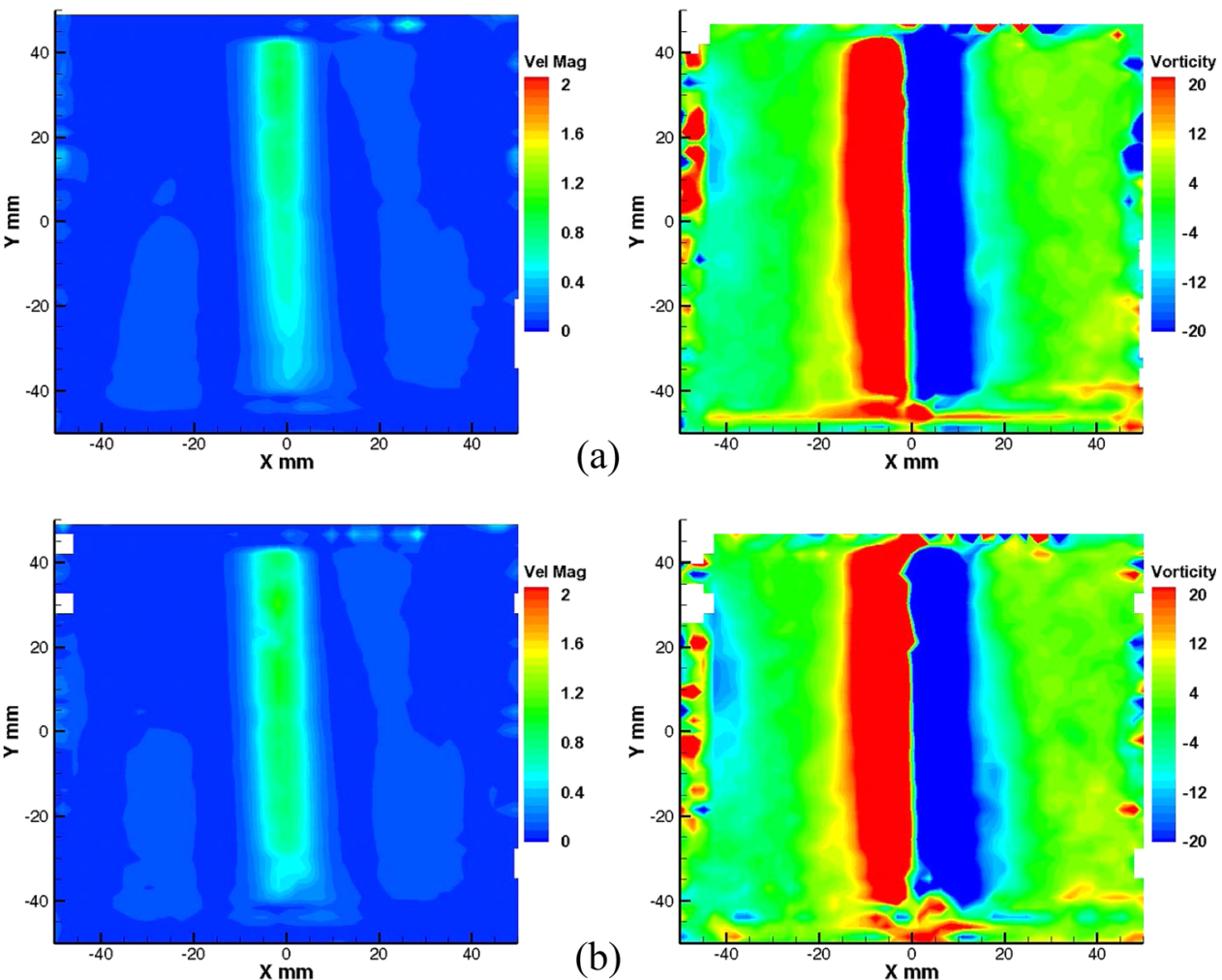

(b)
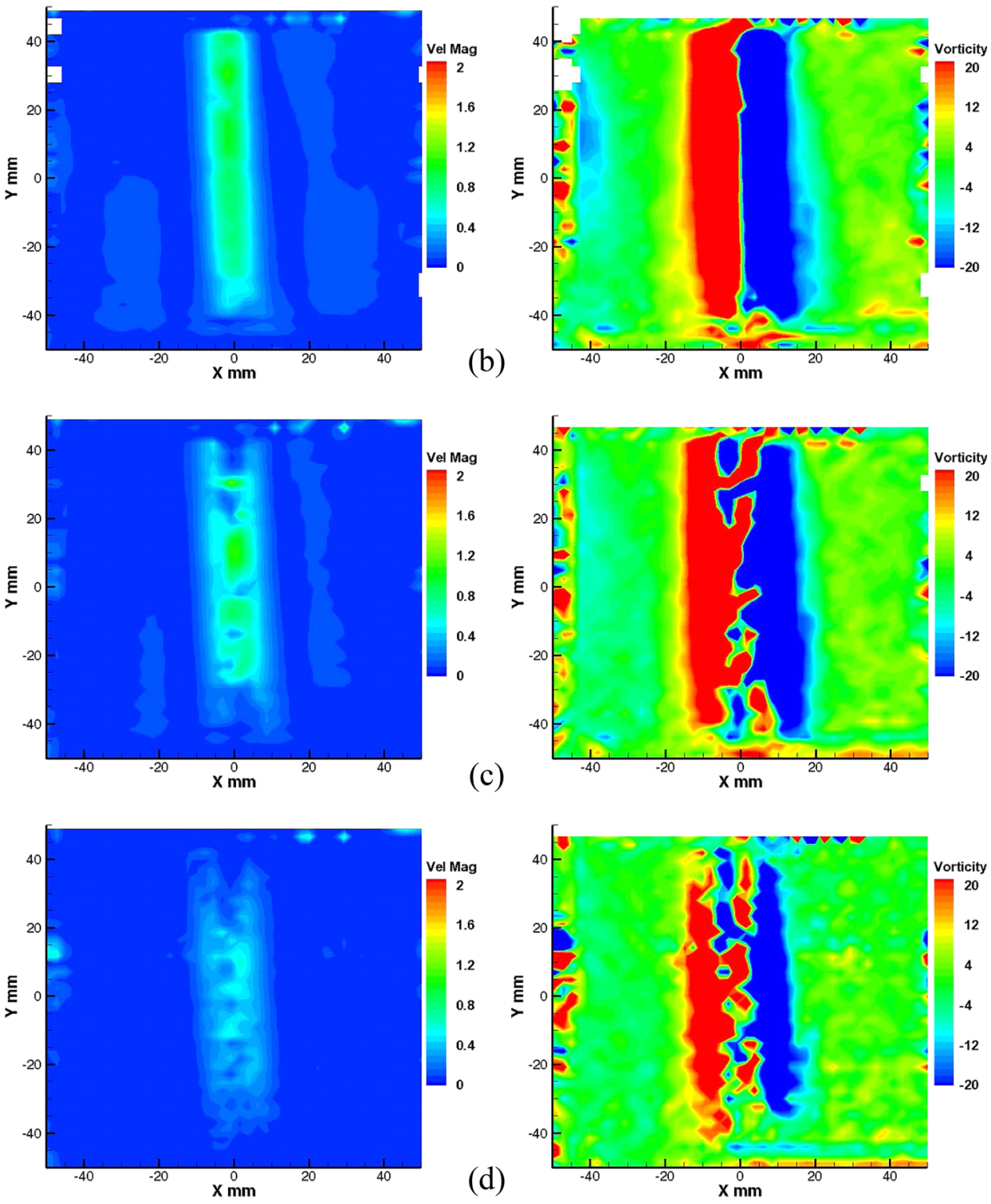

Fig. 17 Averaged magnitude contours of axial velocity and corresponding vortices according to different wall screen flow rate with respect to CFD settings: (a) $50 \%$, (b) $75 \%$, (c) $125 \%$, and (d) $150 \%$

031003-10 / Vol. 135, AUGUST 2013

Transactions of the ASME 

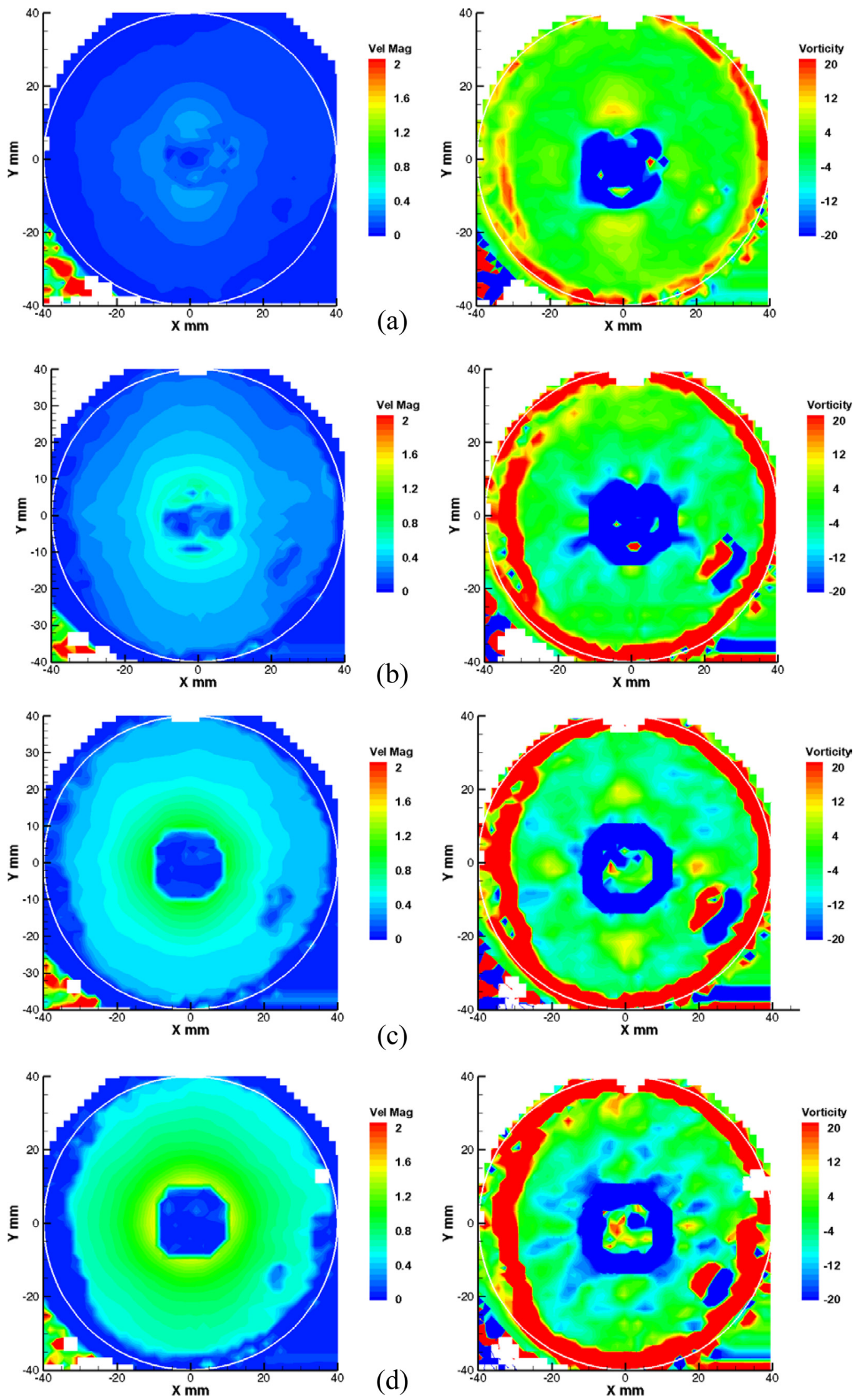

Fig. 18 Magnitude contours of tangential velocity and corresponding vortices, according to different main flow rate with respect to CFD settings: (a) $50 \%$, (b) $75 \%$, (c) $100 \%$ (CFD settings), (d) $125 \%$, and (e) $150 \%$ 

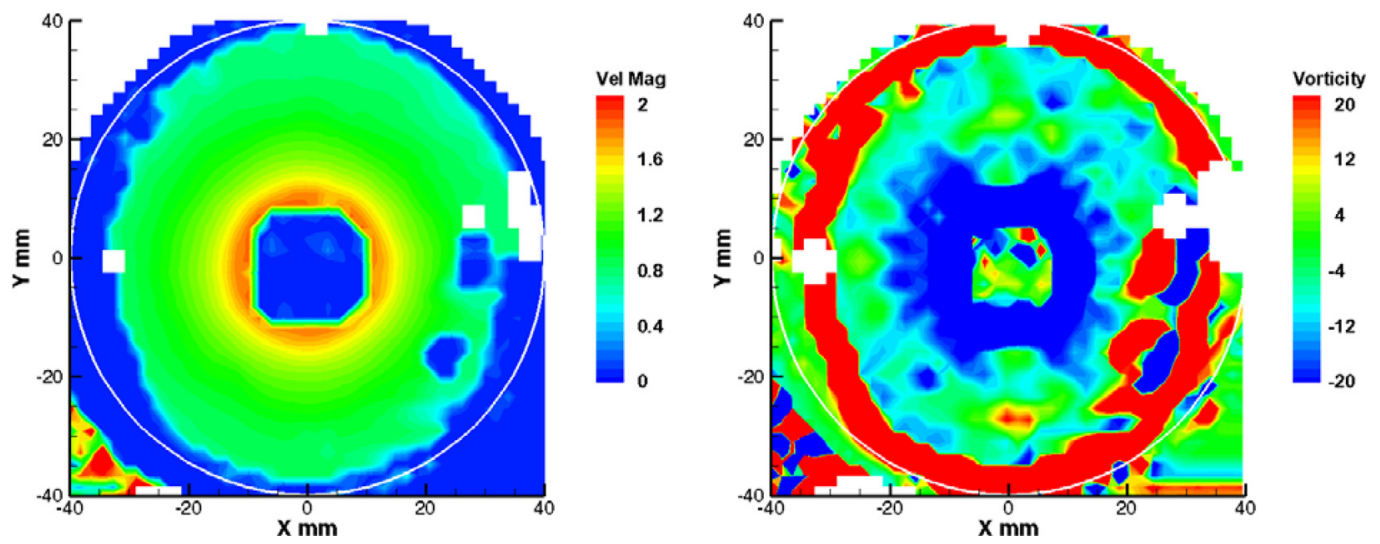

Fig. 18 Continued

The four cases of increasing and decreasing the main flow and wall screen gas by $25 \%$, respectively, with respect to the CFD settings are shown in the figures. The result shows that when the main flow was decreased by $25 \%$, the vertical velocity components became weaker; the vertical vortices along the vortex line became significant in the core region, which produced a stable structure in that region. In comparison, when the wall screen flow was increased by $25 \%$, the vertical vortices along the vortex line became stronger, and the vortices in the wall region grew larger, which implied a relatively more chaotic structure due to the increase of system momentum compared to the CFD settings. When the wall screen flow was decreased by $25 \%$, the vertical vortices along the vortex line became stable and thus defined a clear path for the particle moving upward in the core region. On the other hand, a stronger chaotic structure was identified when the wall screen flow was increased by $25 \%$. Based on the previous observations, either the main flow or the wall screen flow decreased. The results showed a similar tendency of stable vertical vortices and velocity distribution which included less chaotic structure.

To further understand the effect of the flow rate change, cases with $\pm 50 \%$ flow rate changes were performed. When main flow was increased by $50 \%$, the flow became a cyclone-dominated structure, which contained a significant distribution of the system momentum. On the other hand, the flow was wall-screen-flowdominated showing more disturbance in the core region when the wall screen flow was increased by $50 \%$. There was an exception when the main flow rate was decreased to a certain extent. When the main flow was decreased by $50 \%$, the flow became a flow without specific flow structure, which has no specific tendency of velocity or vortices directions. When the wall screen flow was decreased by $50 \%$, the flow showed a series of significant vortices
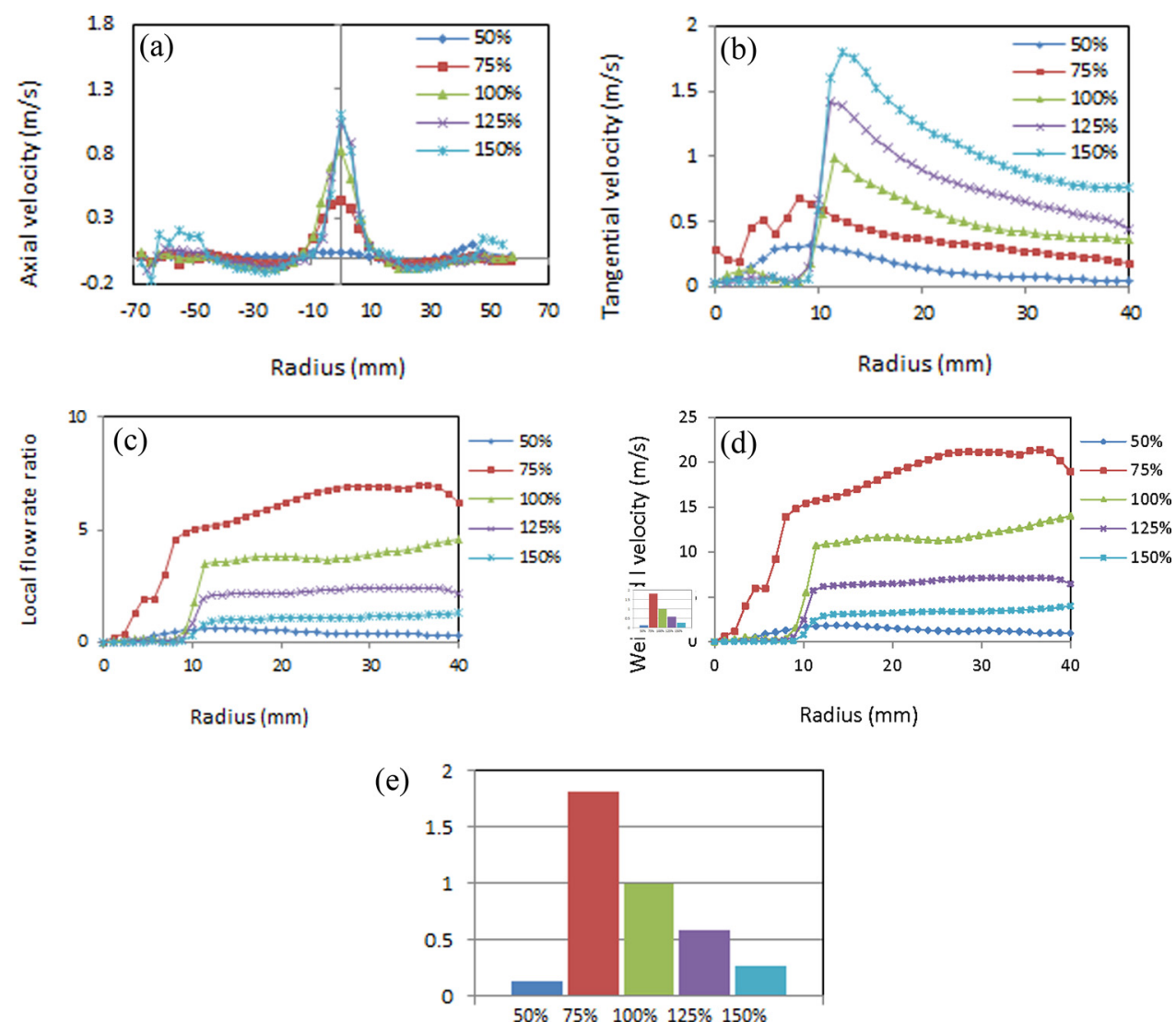

Fig. 19 (a)-(c) Velocity distributions across radius according to different main flow rate. (d) Weighted velocity. (e) Normalized swirl number integrated from Fig. 19(c). 
along the vortex line. This implied that the vortices along the vortex line might not only be dominated by the wall screen flow but also be affected by the main flow that induced the cyclone structure flow. In short, as long as the main flow or the wall screen flow rate increased relative to the CFD settings, the flow field immediately became a more chaotic flow structure.

Since the change of main flow has a major impact on the fluid behavior, an axial cross section of the flow field was captured and subjected to the main flow variation shown in Fig. 18. In the vorticity field of Fig. 18, the opposite direction of vorticity indicated the wall effect though the boundary of the image was at locations between the wall and axis instead of right on the boundary of the wall.

\section{Analysis of Experimental Results}

The effectiveness of the reactor can be increased by increasing the residence time of the gas. This prolonged resident time of the particles in the reactor can be achieve by either reducing the inlet volumetric flow rate or generating stronger vortices that keep the particles in the reactor for a longer time. The ratio of circulating flow rate to the axial flow rate was evaluated to help quantify the resident time. In order to obtain the circulating flow rate, the tangential velocity vectors across the diameter of the reactor from top view images were extracted. The same methodology was used to calculate total axial flow rate from vector field of side view images. Considering error of the radius effect in obtaining local axial flow rates near the wall region, only the most credible part of the velocities used to calculate the total axial flow rate. It was assumed that the axial velocities in the wall region sum up to zero. Note that since this assumption was made, the credible total axial flow rate was not exactly equal to total axial flow rate. Figure 19 shows results of the axial and tangential velocities obtained from Figs. 16 and 18.

Figure 19(a) shows the axial velocity distribution across the reactor diameter, the $\mathrm{x}$-axis represents the radius across the flow field. In Fig. 19(a), the negative velocity between axis and wall regions shows the evidence of a reversing flow which acted as a supplying source to maintain the vortex structure. Figure 19(b) shows tangential velocity distribution captured from the center to the $40 \mathrm{~mm}$ radius, which was the size of the reactor window. The profile possesses a classic Rankine vortex profile with a forced vortex around the axis due to viscous effect and a free vortex tendency due to conservation of angular momentum. Figure 19(c) shows the local tangential-to-axial flow rate ratio defined by following equation

$$
\text { Local tangential-to-axial flow rate ratio }=\frac{r_{i} \times w_{i}}{\sum\left(r_{i} \times v_{i}\right)}
$$

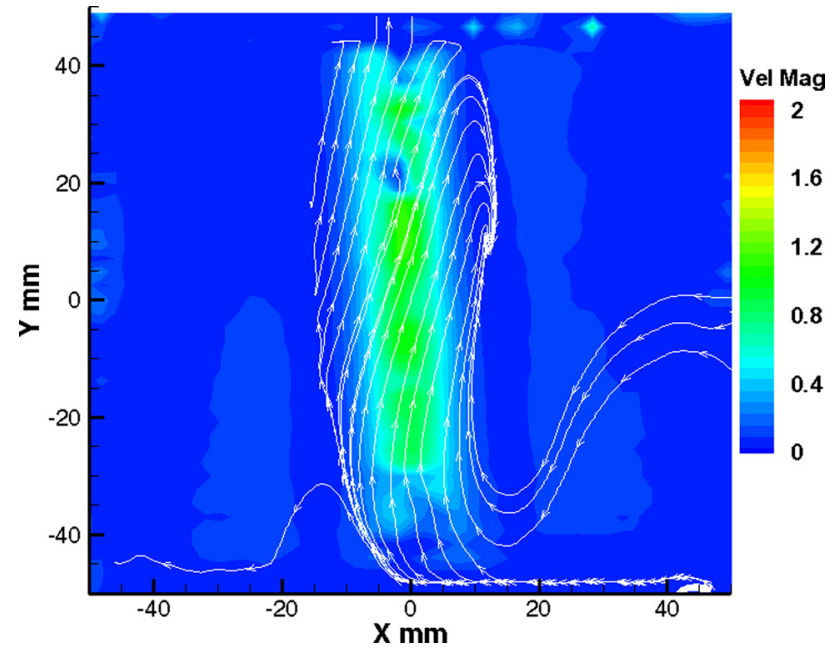

Fig. 20 Stream line distribution of PIV image

where $r$ is the radius, $w$ is the tangential velocity, and $v$ is the axial velocity. It was found that the $75 \%$ main flow case had largest swirling tendency compared to other cases across the whole measuring range. Figure $19(d)$ represents the overall flow rate ratio integrated across the radius and then normalized with the case of CFD setting. By comparing the result in Fig. $19(d)$, the ratio of flow rates shows that at $75 \%$ main flow rate, the cyclone flow could retain the particle in the reactor longer. In the $150 \%$ main flow rate case, a large axial flow rate led to a low flow rate ratio. In the $50 \%$ main flow case, the weak tangential velocity produced a low ratio too. When a Gaussian three point peak estimator was applied to Fig. 19(d), the result showed that the largest ratio takes place at $82 \%$ of main flow rate compared to the CFD settings.

At the core region of the image it is seen that the direction of circulation of the cyclone is the same as the axial flow direction as designed since the streamlines at left bottom of core region were denser compared to the right bottom shown in Fig. 20. At the top of the core region, the path lines were sparse at the top left compared to the top right, indicating the flow was a twisting cyclone according to the flow geometry though the volume variation could locally alter the flow structure.

To determine whether the ratio of flow rates varies as the flow evolves along the vortex line, the tangential velocities on eight planes were captured in an axial direction of the reactor shown in Fig. 21.

The result shows that the velocity profiles between the core and the wall region overlap into a single profile. The whole tangential

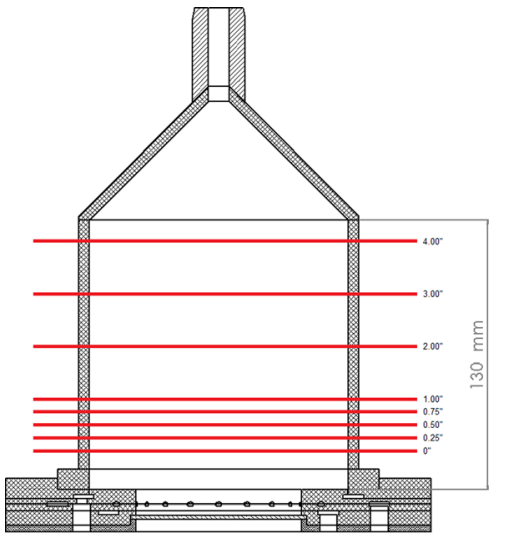

(a)

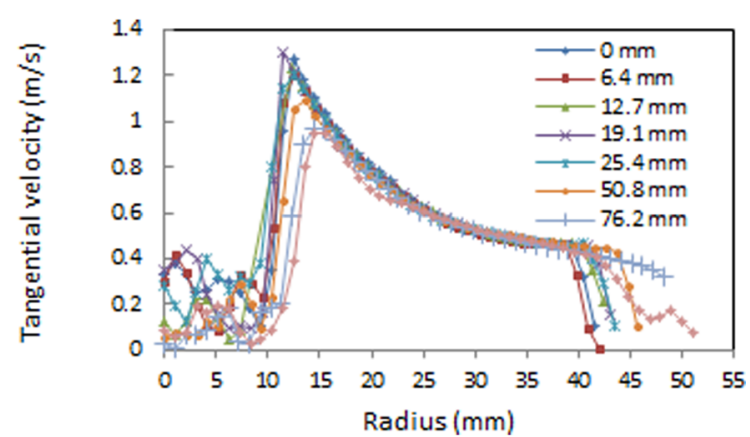

(b)

Fig. 21 Axial evolution of flow field, (a) capture cross sectional planes, (b) tangential velocities distribution versus radius corresponding to cross sectional planes 
velocity field acts as a Rankine vortex with highest speed around the axis decaying toward the wall. The maximum swirling speed and size of the core region were proportional to the axial evolution distance. The tendency of overlapping velocity magnitudes in the region between core region and wall region indicates that the axial evolution of tangential flow rate is dominated by the fluid in the core region. In other word, the variation of tangential flow rate due to axial evolution is negligible. Due to the pin-hole effect, the measuring range of the flow field enlarged proportional to the evolution distance. The decaying and fluctuating velocities within the core region implied a viscous and chaotic out-of-plane viscous fluid motion.

\section{Uncertainty Analysis}

An uncertainty analysis was performed according to the procedures documented by Nishio [24]. The velocity measurement of PIV can be described by the following equation [24] $u=(\Delta X / \Delta t) /$ $M+\delta u$, where $\Delta X$ was the particle displacement, $\Delta t$ was the time gap between an image pair, $M$ was the magnification factor. The flow field range was $146 \times 146 \mathrm{~mm}^{2}$ without considering the local distortion due to curvature on the reactor wall. The average vortex flow speed was $0.5 \mathrm{~m} / \mathrm{s}$ according to the result of PIV measurement. The diameter of the seeding particles was set to $1 \mu \mathrm{m}$ assuming $10 \%$ deviation. The time interval was $150 \mu$ s to reduce pairing loss. A pinhole effect was taken into consideration according to the 2D PIV experimental setup. The maximum combined uncertainty of velocity was about $20 \%$ of the averaged flow speed due to both systematic errors and measurement errors. The secondary combined uncertainties came from the particle displacement were $0.69 \mathrm{~mm}$ for side view capture and $0.68 \mathrm{~mm}$ for top view capture. The combined uncertainty due to time interval is less than $0.1 \%$ for both cases.

\section{Conclusions}

A novel solar reactor concept which has a cyclone in the center and a laminar "wall screening" flow on the walls was introduced as a solution to reduce the carbon deposition problem. A thorough description about the evolution of this solar reactor concept was given based on the flow pattern, temperature contours, and carbon deposition via CFD analysis. It was observed that by appropriate selection of parameters, the concept of aero-shielded solar cyclone reactor can be an attractive option to overcome the problem of carbon particle deposition at the walls and exit.

An experimental evaluation of a prototype reactor was presented and the flow dynamics inside the reactor was analyzed via PIV. In order to create the flow field with fluid dynamics similar to the real case, the pressures and volume flow rates were maintained. Considering the material limitation, heat insulation, and potential danger of using working gas species of the real case such as methane and hydrogen, a work progress was set with atmospheric air. A cyclone structure flow, which increases the residence time and reduces the carbon deposition according to our CFD studies was experimentally achieved. A Rankine-vortex-like fluid motion in the core region was identified. The spatial evolution of the cyclone flow field was quantified. The effects of the volume flow rate to the reactor efficiency was experimentally extracted and calculated. The optimal operating point was compared with the CFD prediction and led to a deviation of $-18 \%$ main flow rate. In Kogan's work [17], a reversing flow between the core region and wall region to feed the vortex motion was observed. In this research, the PIV image also captured and quantified this phenomenon. Decreasing either the main flow or the wall screen flow gave similar tendency of stable vorticity and velocity distribution which included less chaotic structure. On the contrary, increase of the main flow made the flow field a stronger vortex. However, increase of the wall screen flow rate made the vortex start to collapse and become a more chaotic flow structure.

In order to identify the feasibility of using liquid particles (TEG) instead of nanoscale solid carbon to conduct the experi- ment, a particle analysis was performed. The analysis showed that the Stokes number of the liquid particle was acceptable in this application while the amplitude ratio was larger than $99 \%$. According to the density estimation of the solid carbon particles, the density was about two times larger than the liquid particles. However, the diameter of the solid carbon was at least two orders smaller than that of the liquid particle, thus the Stokes number of the carbon particles was even smaller than that of the liquid particles. This result implied that it was possible to trace the liquid particles and consider the particle path same as that of the carbon particles. In future work, it is planned to conduct experiments with solid particles and different flow configurations to achieve optimum cyclone and wall screening.

\section{Acknowledgment}

This publication was made possible by NPRP grant no. (09671-2-255) from the Qatar National Research Fund (a member of Qatar Foundation). The statements made herein are solely the responsibility of the authors.

\section{References}

[1] Ozalp, N., 2008, "Energy and Material Flow Models of Hydrogen Production in the U.S. Chemical Industry," Int. J. Hydrogen Energy, 33, pp. 5020-5034.

[2] Ozalp, N., Kogan, A., and Epstein, M., 2009, "Solar Decomposition of Fossil Fuels as an Option for Sustainability," Int. J. Hydrogen Energy, 34(2), pp. 710-720.

[3] Elizade, I., Rodriguez, M. A., and Ancheyta, J., 2009, "Application of Continuous Kinetic Lumping Modeling to Moderate Hydrocracking of Heavy Oil," Appl. Catal., A, 365, pp. 237-242.

[4] Ancheyta, J., Rana, M. S., and Furimsky, E., 2005, "Hydroprocessing of Heavy Petroleum Feeds: Tutorial," Catal. Today, 109, pp. 3-15.

[5] Castaneda, L. C., Munoz, J. A. D., and Ancheyta, J., 2011, "Comparison of Approaches to Determine Hydrogen Consumption During Catalytic Hydrotreating of Oil Fractions," Fuel, 90(12), pp. 3593-3601.

[6] Rodat, S., Abanades, S., and Flamant, G., 2011, "Co-Production of Hydrogen and Carbon Black From Solar Thermal Methane Splitting in a Tubular Reactor Prototype," Sol. Energy, 85(4), pp. 645-652.

[7] Maag, G., Rodat, S., Flamant, G., and Steinfeld, A., 2010, "Heat Transfer Model and Scale-Up of an Entrained-Flow Solar Reactor for the Thermal Decomposition of Methane," Int. J. Hydrogen Energy, 35(24), pp. 13232-13241.

[8] Muradov, N., Smith, F., Bockerman, G., and Scammon, K., 2009, "Thermocatalytic Decomposition of Natural Gas Over Plasma-Generated Carbon Aerosols for Sustainable Production of Hydrogen and Carbon," Appl. Catal., A, 365(2), pp. 292-300.

[9] Dahl, J. K., Buechler, K. J., Weimer, A. W., Lewandowski, A., and Bingham, C., 2004, "Solar-Thermal Dissociation of Methane in a Fluid-Wall Aerosol Flow Reactor," Int. J. Hydrogen Energy, 29(7), pp. 725-736.

[10] Huang, C., Yao, W., T-Raissi, A., and Muradov, N., 2011, "Development of Efficient Photoreactors for Solar Hydrogen Production," Sol. Energy, 85(1), pp. $19-27$.

[11] Kogan, A., Israeli, M., and Alcobi, E., 2007, "Production of Hydrogen and Carbon by Solar Thermal Methane Splitting. IV. Preliminary Simulation of a Confined Tornado Flow Configuration by Computational Fluid Dynamics," Int. J. Hydrogen Energy, 32(18), pp. 4800-4810.

[12] Trommer, D., Hirsch, D., and Steinfeld, A., 2004, "Kinetic Investigation of the Thermal Decomposition of CH4 By Direct Irradiation of a Vortex-Flow Laden With Carbon Particles," Int. J. Hydrogen Energy, 29(6), pp. 627-633.

[13] Kogan, A, and Kogan, M., 2002, "The Tornado Flow Configuration-An Effective Method for Screening of a Solar Reactor Window," ASME J. Sol. Energy Eng., 124, pp. 206-214.

[14] Hirsch, D., and Steinfield, A., 2004, "Solar Hydrogen Production by Thermal Decomposition of Natural Gas Using a Vortex-Flow Reactor," Int. J. Hydrogen Energy, 29, pp. 47-55.

[15] Ozalp, N., and Jayakrishna, D., 2010, "CFD Analysis of Multi-Phase Turbulent Flow in a Solar Reactor for Emission-Free Generation of Hydrogen," Chem. Eng. Trans., 21, pp. 1081-1086.

[16] Ozalp, N., and Jayakrishna, D., 2010, "CFD Analysis on the Influence of Helical Carving in a Vortex Flow Reactor," Int. J. Hydrogen Energy, 35, pp $6248-6260$.

[17] Kogan, A., Kogan, M., and Barak, S., 2004, "Production of Hydrogen and Carbon by Solar Thermal Methane Splitting. II. Room Temperature Simulation Tests of Seeded Solar Reactor," Int. J. Hydrogen Energy, 29, pp. $1227-1236$.

[18] Ozalp, N., and Kanjirakat, A., 2010, "Lagrangian Characterization of MultiPhase Turbulent Flow in a Solar Reactor for Particle Deposition Prediction," Int. J. Hydrogen Energy, 35, pp. 4496-4509.

[19] Ozalp, N., 2011, "An Overview of Solar Thermal Cracking of Natural Gas: Challenges and Solutions Towards Commercialization," 8th International 
Conference on Heat Transfer, Fluid Mechanics, and Thermodynamics (HEFAT 2011), Republic of Mauritius, July 11-13, Paper No. 1569441867.

[20] Shilapuram, V., Jayakrishna, D., and Ozalp, N., 2011, "Residence Time Distri bution Analysis of Aero-Shielded Solar Cyclone Reactor for Emission-Free Generation of Hydrogen," Int. J. Hydrogen Energy, 36, pp. 13488-13500.

[21] Keane, R. D., and Adrian, R. J., 1991, "Optimization of Particle Image Velocimetry: II. Multiple Pulsed Systems," Meas. Sci. Technol., 2, pp. 963-974.
[22] Adrian, R. J., 1997, "Dynamic Ranges of Velocity and Spatial Resolution of Particle Image Velocimetry,” Meas. Sci. Technol., 8, pp. 1393-1398.

[23] Hart, D. P., 1998, "PIV Error Correction, Experiments in Fluids," 9th International Symposium on Applications of Laser Techniques to Fluid Mechanics, Lisbon, Portugal, July 13-16.

[24] Nishio, S., 2008, "Uncertainty Analysis and Example for PIV Measurements," Proceedings of 25th International Towing Tank Conference (ITTC), Fukuoka, Japan, September 14-20. 